\title{
The usefulness of point-of-care (POC) tests in screening elevated glucose and ketone body levels postmortem
}

\section{Walta, Anna-Mari}

2016-09

Walta , A-M , Keltanen , T , Lindroos , K \& Sajantila , A 2016, ' The usefulness of point-of-care (POC) tests in screening elevated glucose and ketone body levels postmortem ' , Forensic Science International , vol. 266 , pp. 299-303 . https://doi.org/10.1016/j.forsciint.2016.06.003

http://hdl.handle.net/10138/228780

https://doi.org/10.1016/j.forsciint.2016.06.003

publishedVersion

Downloaded from Helda, University of Helsinki institutional repository.

This is an electronic reprint of the original article.

This reprint may differ from the original in pagination and typographic detail.

Please cite the original version. 


\title{
The usefulness of point-of-care (POC) tests in screening elevated glucose and ketone body levels postmortem
}

\author{
Anna-Mari Walta *, Terhi Keltanen, Katarina Lindroos, Antti Sajantila* \\ Laboratory of Forensic Biology, Department of Forensic Medicine, University of Helsinki, P.O Box 40, 00014, Helsinki, Finland
}

\section{A R T I C L E I N F O}

\section{Article history:}

Received 14 March 2016

Received in revised form 21 May 2016

Accepted 4 June 2016

Available online 14 June 2016

\section{Keywords:}

Urine stick test

Blood glucose monitoring device

Postmortem

Hyperglycemia

Ketoacidosis

\begin{abstract}
A B S T R A C T
The aim was to evaluate the performance of point-of-care (POC) tests in detecting glucose and ketone bodies in postmortem (PM) samples and to assess the usefulness of POC tests in sample screening for more precise analyses. Glucose and ketone body, $\beta$-hydroxybutyrate (BHB), were measured from vitreous humor ( $\mathrm{VH})$ in 52 autopsy cases with a POC blood glucose monitoring device (BGMD). In addition glucose and ketone bodies, acetone (Ac) and acetoacetate (AcAc), were measured from urine samples in another set of 59 cases with semi-quantitative stick tests. The results were compared to the concentration in $\mathrm{VH}$ measured with validated methods (values $\geq 7 \mathrm{mmol} / \mathrm{l}$ indicate possible hyperglycemia and total ketone body levels $\geq 3 \mathrm{mmol} / \mathrm{l}$ ketoacidosis). The sensitivity for glucose with the BGMD was 1.0 and specificity 0.94 when the threshold value for the meter to predict elevated glucose was set to $\geq 10 \mathrm{mmol} / \mathrm{l}$. The correlation between the BGMD and the validated method was strong $\left(R^{2}=0.89\right)$. For detecting ketoacidosis, the BGMD had a sensitivity of 1.0 and specificity of 0.73 , when the threshold value was set to $2.5 \mathrm{mmol} / \mathrm{l}$. The urine stick test presented a sensitivity of 0.89 and specificity of 0.90 for detecting elevated VH glucose concentration. The sensitivity and specificity for the stick test to detect cases with possible ketoacidosis were 0.84 and 0.68 , respectively. According to the results, BGMD can be reliably applied for sample screening, although more samples need to be analyzed for delineating the correct threshold values. In the case of glucose, the urine stick tests could be indicative in detecting cases with $\mathrm{VH}$ glucose $\geq 10 \mathrm{mmol} / \mathrm{l}$. For predicting possible ketoacidosis with elevated $\mathrm{VH}$ total ketone bodies, the stick test is not reliable as the test presented both false-positive and -negative results.
\end{abstract}

(c) 2016 Elsevier Ireland Ltd. All rights reserved.

\section{Introduction}

Diabetic ketoacidosis (DKA) and hyperosmolar hyperglycemia (HHS) are acute, life-threatening metabolic complications caused by poorly or untreated diabetes (DM). In both conditions, blood glucose levels are elevated as a result of lack of insulin or impaired effects of it. In DKA concomitant enhanced release of counter regulatory hormones (glucagon, catecholamines, cortisol and growth hormone) leads to lipolysis and to the production of ketone bodies acetone (Ac), acetoacetate (AcAc) and $\beta$-hydroxybutyrate (BHB) followed by acidosis $[1,2]$.

Self-monitoring of diabetes is recommended as part of routine care for all diabetic patients to assess their glycemic control [3]. Traditional home tests include e.g. semi-quantitative

\footnotetext{
* Corresponding author.

E-mail addresses: annamari.walta@gmail.com (A.-M. Walta), antti.sajantila@helsinki.fi (A. Sajantila).
}

stick tests for the detection of glucose and ketone bodies in urine or blood. The ketone testing is based on the Legaĺs reaction in which Ac and AcAc in the sample react in the presence of alkali with nitroprusside (nitroferricyanide) and glycine to produce a colored reaction on a test strip [4], while glucose can be detected in a glucose specific oxidase-peroxidase based reaction [5]. Urine results are proportional to blood levels, but the results are affected e.g. by fluid intake and urine concentration. The results do not reflect the situation at the time of the measurement because the urine could have been in the bladder for many hours [6].

Blood testing with electrochemical monitoring devices, in which the reaction between the measured analyte and the chemicals on the test strip produces an electric current with varying intensities depending on the amount of the analyte in the sample, provide more accurate results [6-8]. For the evaluation of possible ketoacidosis, the electrochemical devices have an important improvement compared to the stick tests, as they detect BHB, the main ketone body in ketoacidosis [9]. 
Ketone bodies and glucose can be measured postmortem (PM) in combination with other biochemical markers (e.g. glycated hemoglobin, HbA1c) to assess antemortem (AM) hyperglycemia and ketoacidosis. These conditions may not be easily detected by other methods in the autopsy $[10,11]$. Vitreous humor $(\mathrm{VH})$ is often preferred as a sample material over blood as it is less affected by putrefaction and biochemical PM changes e.g. glycolysis [12] and the concentrations correlate with blood levels $[13,14]$. The VH glucose concentration is considered to be elevated when it exceeds 6.9-10 $\mathrm{mmol} / \mathrm{l}$ (the threshold depending on the study) $[11,15,16]$. For indicating diabetic ketoacidosis PM, the most relevant markers along $\mathrm{VH}$ glucose are ketone bodies and HbA1c [11,15]. The analyses performed are generally highly accurate and somewhat costly as they involve sophisticated methods like gas chromatography. In this study, we wanted to evaluate the performance of different point-of-care (POC) tests to simplify the detection of glucose and ketone bodies in PM samples. Urine concentrations of glucose, Ac and AcAc, were evaluated with stick test. Glucose and $\mathrm{BHB}$ in $\mathrm{VH}$ were measured with POC blood glucose electrochemical monitoring system. The ultimate aim of our study was to assess the usefulness of POC tests in pre-screening the samples for more precise, but costly laboratory analyses.

\section{Materialand methods}

\subsection{Forensic autopsy samples}

To evaluate the performance of urine stick tests to semiquantitate urinary glucose and Ac/AcAc in PM samples urine was collected from 59 cases. VH samples were attained from another set of 52 autopsies to test the performance of the BGMD in quantitation of glucose and BHB. The results were compared to the results attained by analyzing $\mathrm{VH}$ with validated methods. The study was approved by the National Institute of Health and Welfare and Helsinki University Central Hospital Ethical Committee.

\subsection{Evaluation of urine glucose and Ac/AcAc}

Urine specimens were collected in a tube without preservative in the autopsy. The test strip (Keto-Diabur-Test 5000, Roche) was briefly immersed in the urine. Reading range for glucose was from $5.6-278 \mathrm{mmol} / \mathrm{l}$. For Ac/AcAc (+) $0.5-4 \mathrm{mmol} / \mathrm{l}$ referred slightly increased (++) 4-10 mmol/l increased and $(+++)>10 \mathrm{mmol} / \mathrm{l}$ highly increased concentration. The readings were observed after 2 min according to manufacturer's recommendation.

\subsection{Glucose and BHB measurements from VH with BGMD}

Glucose and BHB concentrations in $\mathrm{VH}$ were measured with FreeStyle Precision Neo blood glucose monitoring system (Abbott, Diabetes Care Ltd, UK) using Freestyle Precision blood glucose and $\beta$-ketone test strips. For both measurements, $60 \mu \mathrm{l}$ of vitreous humor sample was centrifuged at $5000 \times \mathrm{g}$ for $5 \mathrm{~min}$. The sample amount used was $\sim 3 \mu$ l of supernatant. The measurements were performed according to the manufacturer's instructions. The measuring range for glucose was 1.1-27.8 mmol/1 and for BHB $0-8 \mathrm{mmol} / \mathrm{l}$.

\subsection{Glucose and total ketone body measurements from $\mathrm{VH}$ with validated methods}

Reference levels for glucose were measured from perchloric acid (PCA) precipitated VH samples with validated enzymatic hexokinase/glucose-6-phosphate dehydrogenase method as previously described [17]. In brief, glucose in the sample is phosphorylated to glucose-6- phosphate (G6P) by hexokinase. G6P is then oxidized with concurrent reduction of cofactor NADP to $\mathrm{NADPH}$. The amount of NADPH is detected as a change of absorption with $340 \mathrm{~nm}$ wavelength and it is directly proportional to the amount of glucose in the sample. Values $\geq 7 \mathrm{mmol} / \mathrm{l}$ were considered to be elevated.

Reference levels for total ketone bodies were measured with analytical total ketone body method, described by Felby \& Nielsen [18]. BHB is first enzymatically converted to AcAc, which is decarboxylated to Ac by heating. The Ac is subsequently measured with headspace gas-chromatography (HS-GC). The HS-GC system used was PerkinElmer TurboMatrix-HS connected to a PerkinElmer Clarus 500 GC with flame ionization detector (FID) (PerkinElmer Inc., USA). Values $\geq 3 \mathrm{mmol} / \mathrm{l}$ are considered to indicate ketoacidosis.

\section{Results}

\subsection{Urine analysis}

The correspondence between glucose urine stick test results and glucose concentration measured from $\mathrm{VH}$ are presented in Table 1 . The sensitivity for the stick test to detect cases with elevated $\geq 7 \mathrm{mmol} / \mathrm{l} \mathrm{VH}$ glucose levels was 0.89 and the specificity 0.90 when the threshold value was set to negative versus any positive results. As for the stick test detecting cases with elevated $\geq$ $3 \mathrm{mmol} / \mathrm{l} \mathrm{VH}$ total ketone bodies, the sensitivity was 0.84 and the specificity 0.68 (Table 2 ).

\subsection{Glucose concentrations in VH measured with BGMD compared to the validated method}

The results of sensitivity and specificity of the BGMD for predicting elevated $\mathrm{VH}$ glucose $(\geq 7 \mathrm{mmol} / \mathrm{l})$ are presented in Table 3 . With the threshold value of $\geq 7 \mathrm{mmol} / \mathrm{l}$, the sensitivity and specificity for the BGMD were 1.0 and 0.69 , respectively. By increasing the threshold value to $\geq 10 \mathrm{mmol} / \mathrm{l}$, the specificity was 0.94 and the sensitivity 1.0. The correlation between the $\mathrm{VH}$ glucose levels measured with the BGMD and the validated method was strong, $R^{2}=0.89(n=46)$ (Fig. 1). The results of BGMD were significantly higher when compared to validated method: $10.8 \mathrm{mmol} / \mathrm{l}$ vs. $7.5 \mathrm{mmol} / \mathrm{l}(p=0.03)$.

The agreement of the methods was compared by using the Bland-Altman plot. The results were $\ln$ transformed before the analysis as the variance increases with increasing concentration [19]. The difference between the glucose concentrations attained with the two methods and the 95\% limits of agreement are represented in Fig. 2. The dashed line shows the level with perfect agreement, visualizing the higher results attained with the BGMD compared to the validated reference method. The $95 \%$ limits of

Table 1

The correspondence between glucose urine stick test results and glucose concentration measured from $\mathrm{VH}$.

\begin{tabular}{lllll}
\hline $\begin{array}{l}\text { Urine glucose } \\
\text { mmol/l }\end{array}$ & $\begin{array}{l}\text { VH glucose } \\
<7 \mathrm{mmol} / \mathrm{l}\end{array}$ & $\begin{array}{l}\text { VH glucose } \\
\geq 7 \mathrm{mmol} / \mathrm{l}\end{array}$ & Sensitivity & Specificity \\
\hline $\begin{array}{l}\text { Negative } \\
5.6\end{array}$ & 45 & 1 & 0.89 & 0.90 \\
14 & 2 & 0 & & \\
28 & 1 & 0 & & \\
56 & 0 & 0 & & \\
111 & 1 & 2 & & \\
167 & 0 & 1 & & \\
278 & 0 & 3 & \\
& 1 & 2 & & \\
& & $(n=50)$ & & \\
\end{tabular}


Table 2

The correspondence between Ac/AcAc stick test results and total ketone bodies measured from $\mathrm{VH}$.

\begin{tabular}{lllll}
\hline $\begin{array}{l}\text { Urine acetone } \\
\text { acetoacetate }\end{array}$ & $\begin{array}{l}\text { Total ketone } \\
\text { bodies } \\
V H<3 \mathrm{mmol} / \mathrm{l}\end{array}$ & $\begin{array}{l}\text { Total ketone } \\
\text { bodies } \\
V H \geq 3 \mathrm{mmol} / \mathrm{l}\end{array}$ & Sensitivity & Specificity \\
\hline $\begin{array}{l}\text { Negative } \\
(1 \mathrm{mmol} / \mathrm{l})+\end{array}$ & 27 & 3 & 0.84 & 0.68 \\
$(5 \mathrm{mmol} / \mathrm{l})++$ & 0 & 11 & & \\
$(15 \mathrm{mmol} / \mathrm{l})+++$ & 0 & 3 & & \\
& $n=40$ & 2 & & \\
& $n=19$ & &
\end{tabular}

Table 3

Sensitivity and specificity for the POC-test to measure glucose concentration in VH.

\begin{tabular}{lllll}
\hline $\begin{array}{l}\text { POC-method, } \\
\text { glucose mmol/l }\end{array}$ & $\begin{array}{l}\text { Reference } \\
\text { method, } \\
\text { glucose } \\
<7 \mathrm{mmol} / \mathrm{l}\end{array}$ & $\begin{array}{l}\text { Reference } \\
\text { method, } \\
\text { glucose } \\
\geq 7 \mathrm{mmol} / \mathrm{l}\end{array}$ & Sensitivity & Specificity \\
\hline$<7$ & 22 & 0 & 1.0 & 0.69 \\
$\geq 7$ & 10 & 19 & & \\
$<10$ & 30 & 0 & 1.0 & 0.94 \\
$\geq 10$ & 2 & 19 & & \\
& $n=32$ & $n=19$ & & \\
\end{tabular}

agreement are wide apart, with the upper limit of 0.6 and the lower limit 1.6, indicating a great variation between the differences and proving that the reference method cannot be directly replaced with the BGMD. For primary screening it is critical to recognize cases with elevated $(\geq 7 \mathrm{mmol} / \mathrm{l}) \mathrm{VH}$ glucose levels. As the mean difference between the methods calculated from the results before In transformation is $\sim-3.3 \mathrm{mmol} / \mathrm{l}$, the cut-off value of $10 \mathrm{mmol} / \mathrm{l}$ for predicting elevated $\mathrm{VH}$ glucose values, proposed already by the sensitivity results, is supported by the Bland-Altman analysis.

\subsection{BHB concentrations in VH measured with BGMD compared to the validated method}

Table 4 presents the sensitivity and specificity for the BGMD in detecting elevated total ketone bodies in $\mathrm{VH}(\geq 3 \mathrm{mmol} / \mathrm{l})$. The sensitivity for BGMD was 1.0 even with different threshold values 1.5 or $2.5 \mathrm{mmol} / \mathrm{l}$. The specificity was at least 0.50 but improved to 0.73 when threshold value was set to $2.5 \mathrm{mmol} / \mathrm{l}$.

\section{Discussion}

\subsection{Urine sticks}

Testing of glucose and ketone bodies in urine is based on the assumption that the urine concentrations roughly correlate with the level in blood. However, the tests present several problems that have to be considered when evaluating the results. In the case of urine glucose testing, the test is impaired by the wide individual variation in renal glucose barrier (approximately $10 \mathrm{mmol} / \mathrm{l}$ ). This threshold value might even increase after a long term DM. Hence, the test result does not give information about the blood concentration below the renal glucose barrier and a negative urine glucose result does not distinguish e.g. mild or moderate hyperglycemia [6]. In forensic setting, high urine glucose levels can be indicative of diabetic coma. But as urine glucose level only weakly correlates with VH glucose concentrations, low levels of glucose in urine does not exclude high vitreous glucose concentrations [10].

In this study, the urine stick test presented a good sensitivity of 0.89 for the estimation of increased $\mathrm{VH}$ glucose values. However, there were only few cases in the group with elevated vitreous glucose concentration $(n=9)$. The single false negative urine result presented slightly elevated $7.4 \mathrm{mmol} / \mathrm{l}$ glucose concentration in $\mathrm{VH}$. In all of the cases with positive urine- and elevated VH result, the VH glucose level exceeded $10 \mathrm{mmol} / \mathrm{l}$ (11.6-25.5 mmol/l). Since VH glucose concentrations have been shown to be

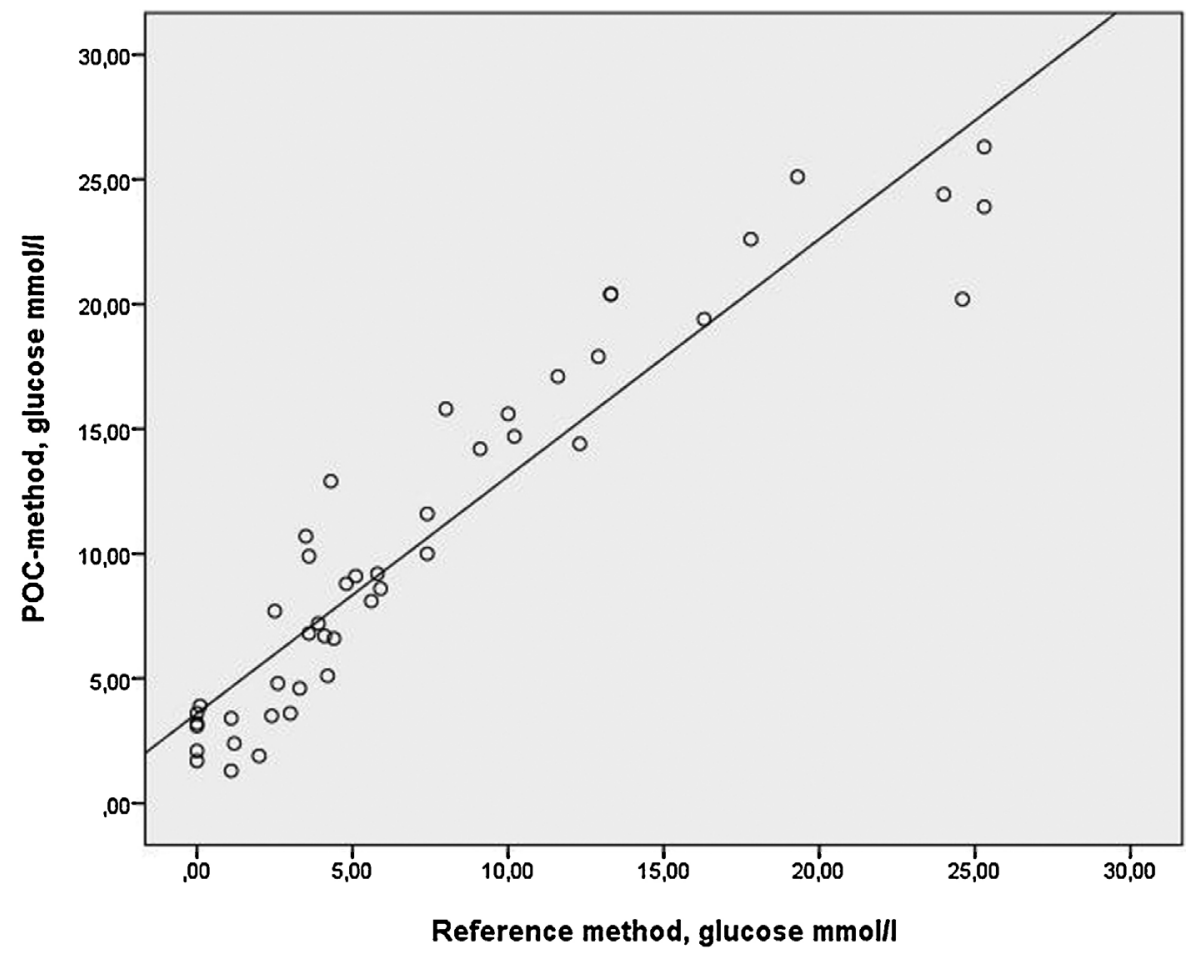

Fig. 1. Correlation of glucose concentration $(\mathrm{mmol} / \mathrm{l})$ in $\mathrm{VH}$ measured with the POC and reference method $(n=46)$. 


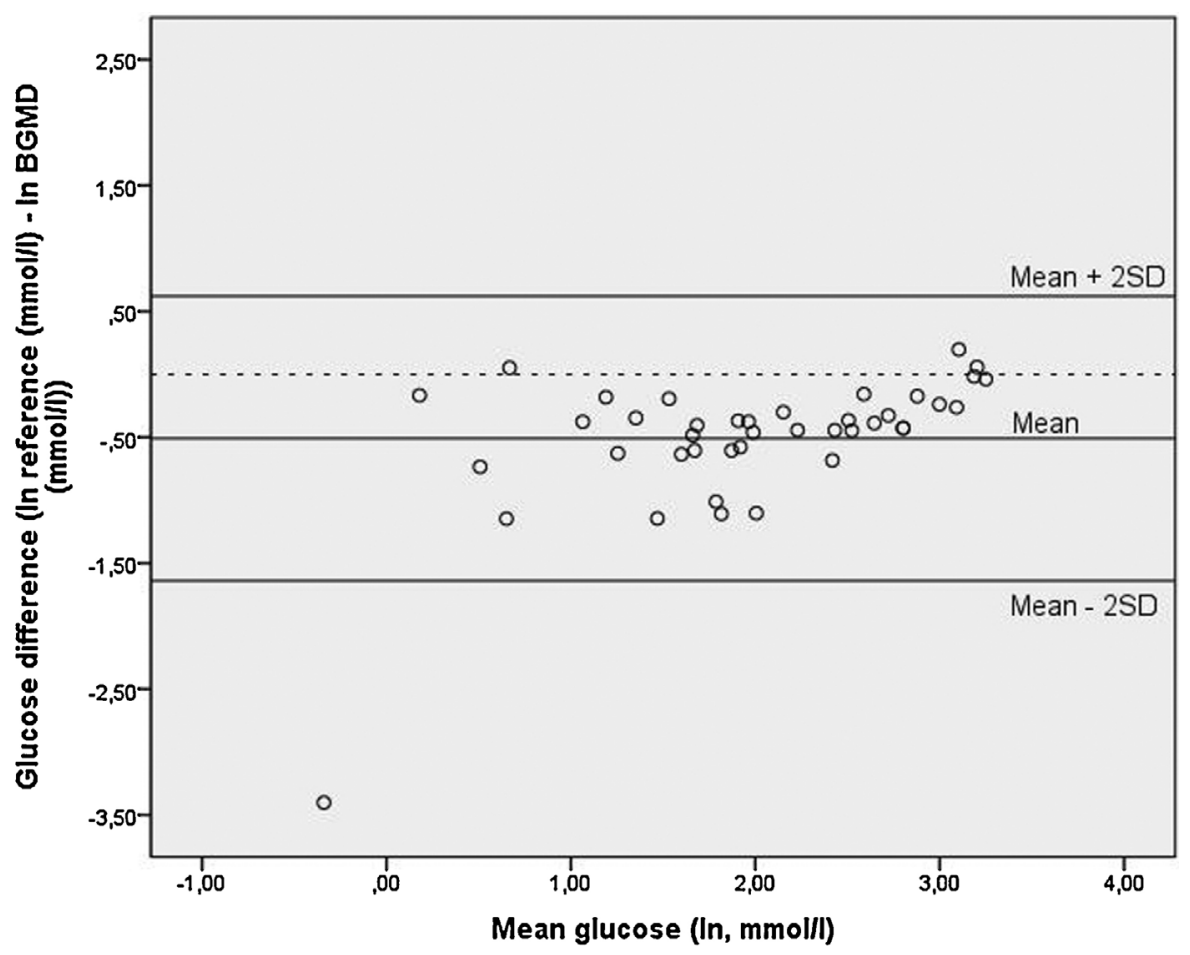

Fig. 2. Glucose difference between the reference method and BGMD, ln transformed data.

Table 4

Sensitivity and specificity for the POC-test to measure $\beta$-hydroxybutyrate in $\mathrm{VH}$.

\begin{tabular}{lllll}
\hline $\begin{array}{l}\beta \text {-hydroxybutyrate } \\
\text { mmol/l }\end{array}$ & $\begin{array}{l}\text { Total ketone } \\
\text { bodies } \\
\text { normal } \\
<3 \mathrm{mmol} / \mathrm{l}\end{array}$ & $\begin{array}{l}\text { Total } \\
\text { ketone } \\
\text { bodies } \\
\text { increased } \\
\geq 3 \mathrm{mmol} / \mathrm{l}\end{array}$ & Sensitivity & Specificity \\
\hline$<1.5$ & 13 & 0 & 1.0 & 0.50 \\
$\geq 1.5$ & 13 & 26 & & \\
$<2.5$ & 19 & 0 & 1.0 & 0.73 \\
$\geq 2.5$ & 7 & 26 & & \\
& $n=26$ & $n=26$ & & \\
\hline
\end{tabular}

approximately half of the concentration in blood [20], the $10 \mathrm{mmol} / \mathrm{l}$ would roughly correspond to $20 \mathrm{mmol} / \mathrm{l}$ in blood, clearly exceeding any renal barrier threshold. The reliability of the stick test is challenged by the false positive results making the test unspecific.

The major pitfall for testing ketone bodies with stick test is that the test detects only Ac and AcAc and, not BHB. In ketoacidosis, $\mathrm{BHB}$ is the main ketone body as the ratio between BHB and AcAc, being normally $\sim 1: 1$, shifts strongly toward BHB [4]. In PM diagnosis of ketoacidosis, AcAc is considered to the have less of an analytical value [15] due to its spontaneous decarboxylation to Ac. Ac itself is a neutral substance and does not contribute to acidosis. It is usually abnormally high in DKA [4] but low or absent levels of Ac do not exclude pathologically significant BHB concentrations [21]. Ac is thought to be indicative mainly of a sustained severe ketoacidosis [22] with extremely elevated total ketone body concentrations [23]. The stick tests detecting only Ac and AcAc are not sufficient to be used for primary screening of ketoacidosis, as milder cases would not be recognized. In this study, the stick test showed both false -positive and -negative results when compared to the HS-GC results from $\mathrm{VH}$, indicating that the test is not reliable for PM sample screening.
The sensitivity and specificity of urine stick tests to be used for the detection of hyperglycemia and ketosis postmortem has previously been studied by Mitchell et al. [24]. In a sample set of 188 forensic autopsy cases their results on urine glucose stick test were somewhat concurrent with ours with a sensitivity of 0.83 and specificity of 0.93 . As for the detection of ketone bodies in urine, the results between the studies differed. A sensitivity attained by Mitchell et al. was 1.0 while the specificity was as low as 0.12 (our results, sensitivity was 0.84 and specificity 0.68 .) A reason for these differences can arise from the interpretation. As Mitchell et al., also we noticed that it is sometimes difficult to precisely define the shade of the color in the stick test when comparing it to the measurement scale. In both studies, this problem was emphasized particularly when the sample urine was of dark color. Additionally, small number of positive results is naturally a limitation of the studies.

\subsection{Blood glucose monitoring device}

For acquiring more accurate results, electrochemical measurement from capillary blood is recommended clinically for both glucose and ketone body determinations [6-8]. In this study, we tested the suitability of one electrochemical device, Freestyle Precision blood glucose monitoring meter, to be used for screening glucose and BHB in PM samples. The results presented that $\mathrm{VH}$ can be used as an alternative sample matrix. The correlation of the glucose results between the BGMD and the reference method was strong, $R^{2}=0.89$. The higher results with the BGMD are probably due to the differences in the sampling process. The samples used in BGMD measurement were undiluted, as in the reference method the samples are diluted in a sample precipitation process. The Bland-Altman analysis revealed a great variation between the differences despite of the strong correlation, proving that the reference method cannot be directly replaced with the BGMD. However as the purpose of this study was to assess the usefulness of POC tests to be used in primary screening, the tendency of BGMD to overestimate the glucose concentration should not be a 
problem. According to the Bland-Altman analysis and the sensitivity/specificity measurement results the threshold value for the BGMD to detect cases with elevated VH glucose could be set to $10 \mathrm{mmol} / \mathrm{l}$, which is also a threshold value suggested by others for PM samples [15,16]. Threshold value of $10 \mathrm{mmol} / \mathrm{l}$ gives a sensitivity of 1.0 and specificity of 0.94 . Nevertheless for delineating the appropriate threshold value, more samples need to be analyzed.

BGMD measures only BHB, not total ketone bodies, like the reference HS-GC method. However, as the BHB is the most important ketone body in ketoacidosis it is reasonable to be analyzed in preliminary screening of the samples. By increasing the threshold value to $2.5 \mathrm{mmol} / \mathrm{l}$, a BHB threshold value proposed by Iten \& Meier [9] for fatal ketoacidosis, the sensitivity is 1.0 and the specificity improves to 0.73 .

\section{Conclusions}

For the sample screening to be useful in PM setting, appropriate threshold value for such screening method is critical. Good sensitivity is somewhat more important than specificity, as it guarantees that samples needing more precise analyses are detected and sent to further testing. However, poor specificity fades the idea of screening, due to unacceptable amount of false positives. The results from this study suggest that POC tests in general and the studied BGMD in particular, can be promising for presumptive assay for the PM samples. It is noteworthy, that the method does not include such cases where the VH glucose concentration is below the threshold value but HbA1c, indicating prolonged high glucose exposure, is elevated. The urinary stick test could possibly be used to detect cases with $\mathrm{VH}$ glucose values $\geq$ $10 \mathrm{mmol} / \mathrm{l}$, but predicting possible ketoacidosis with elevated $\mathrm{VH}$ total ketone bodies, the stick test is not suited according to our study.

\section{References}

[1] M.F. Delaney, A. Zisman, W.M. Kettyle, Diabetic ketoacidosis and hyperglycemic hyperosmolar nonketotic syndrome, Endocrinol. Metab. Clin. North Am. 29 (4) (2000) 683-705.
[2] A.E. Kitabchi, et al., Hyperglycemic crises in patients with diabetes mellitus, Diabetes Care 26 (Suppl 1) (2003) S109-S117.

[3] Standards of medical care in diabetes-2014, Diabetes Care 37 (Suppl 1) (2014) S14-S80.

[4] L. Laffel, Ketone bodies: a review of physiology, pathophysiology and application of monitoring to diabetes, Diabetes Metab. Res. Rev. 15 (6) (1999) 412-426.

[5] A.H. Free, et al., Simple specific test for urine glucose, Clin. Chem. 3 (3) (1957) $163-168$.

[6] D.E. Goldstein, et al., Tests of glycemia in diabetes, Diabetes Care 27 (7) (2004) $1761-1773$.

[7] B. Guerci, et al., Advantages to using capillary blood beta-hydroxybutyrate determination for the detection and treatment of diabetic ketosis, Diabetes Metab. 31 (4 Pt 1) (2005) 401-406.

[8] T.M. Wallace, et al., The hospital and home use of a 30 -second hand-held blood ketone meter: guidelines for clinical practice, Diabet. Med. 18 (8) (2001) 640-645.

[9] P.X. Iten, M. Meier, Beta-hydroxybutyric acid-an indicator for an alcoholic ketoacidosis as cause of death in deceased alcohol abusers, J. Forensic. Sci. 45 (3) (2000) 624-632.

[10] C. Hess, et al., Detection of diabetic metabolism disorders post-mortem-forensic case reports on cause of death hyperglycaemia, Drug Test Anal. 5 (9-10) (2013) 795-801.

[11] J. Hockenhull, et al., Investigation of markers to indicate and distinguish death due to alcoholic ketoacidosis, diabetic ketoacidosis and hyperosmolar hyperglycemic state using post-mortem samples, Forensic Sci. Int. 214 (1-3) (2012) 142-147.

[12] J.I. Coe, Use of chemical determinations on vitreous humor in forensic pathology, Forensic Sci. 17 (4) (1972) 541-546.

[13] D.J. Pounder, R.J. Stevenson, K.K.. Taylor, Alcoholic ketoacidosis at autopsy, J. Forensic Sci. 43 (4) (1998) 812-816.

[14] C. Hess, F. Musshoff, B. Madea, Disorders of glucose metabolism-post mortem analyses in forensic cases: part I, Int. J. Legal Med. 125 (2) (2011) 163-170.

[15] C. Palmiere, et al., Is the formula of Traub still up to date in antemortem blood glucose level estimation? Int. J. Legal Med. 126 (3) (2012) 407-413.

[16] B. Zilg, et al., Postmortem identification of hyperglycemia, Forensic Sci. Int. 185 (1-3) (2009) 89-95.

[17] H. Sippel, M. Mottonen, Combined glucose and lactate values in vitreous humour for postmortem diagnosis of diabetes mellitus, Forensic Sci. Int. 19 (3) (1982) 217-222.

[18] S. Felby, E. Nielsen, Determination of ketone bodies in postmortem blood by headspace gas chromatography, Forensic Sci. Int. 64 (2-3) (1994) 83-88.

[19] J.M. Bland, D.G. Altman, Measuring agreement in method comparison studies, Stat. Methods Med. Res. 8 (2) (1999) 135-160.

[20] O. Lundquist, S. Osterlin, Glucose concentration in the vitreous of nondiabetic and diabetic human eyes, Graefes Arch. Clin. Exp. Ophthalmol 232 (2) (1994) 71-74.

[21] C. Palmiere, M. Augsburger, The postmortem diagnosis of alcoholic ketoacidosis, Alcohol Alcohol. 49 (3) (2014) 271-281.

[22] G.F. Cahill Jr., Ketosis, Kidney Int. 20 (3) (1981) 416-425.

[23] T. Keltanen, et al., Assessment of Traub formula and ketone bodies in cause of death investigations, Int. J. Legal Med. 127 (6) (2013) 1131-1137.

[24] R. Mitchell, S.D. Thomas, N.E. Langlois, How sensitive and specific is urinalysis 'dipstick' testing for detection of hyperglycaemia and ketosis? An audit of findings from coronial autopsies, Pathology 45 (6) (2013) 587-590. 